Chirurg 2017 $\cdot 88: 447$

DOI 10.1007/s00104-017-0428-x

Online publiziert: 20. April 2017

๑) Springer Medizin Verlag GmbH 2017

CrossMark

\section{Originalpublikation}

Denost $Q$, Rouanet $P$, Faucheron J-L et al (2017) To drain or not to drain infraperitoneal anastomosis after rectal excision for cancer: The GRECCAR 5 randomized trial. Ann Surg 265:474-480

Hintergrund. Nach tiefer anteriorer Rektumresektion sollen Drainagen die Ansammlung von Wundsekret in der präsakralen Höhle verhindern und die frühzeitige Erkennung einer Anastomoseninsuffizienz ermöglichen. Zusätzlich kann in manchen Fällen eine Revisionsoperation vermieden werden, wenn die Leckage durch eine einliegende Drainage frühzeitig erfasst und suffizient drainiert wird. Dieser theoretische Nutzen ist jedoch kaum durch prospektive Arbeiten belegt $[2]$.

Methoden. In diese multizentrische, französische Studie wurden zwischen 2011 und 2014 Rektumkarzinompatienten eingeschlossen, die eine Rektumresektion erhielten. Einschlusskriterium war der Erhalt des Sphinkters und die Anlage einer Anastomose unterhalb der peritonealen Umschlagsfalte. Die Patienten wurden in zwei Gruppen randomisiert. Gruppe A erhielt intraoperativ eine Drainage mit Sog, die hinter der Anastomose in die präsakrale Höhle eingelegt wurde, Gruppe B erhielt keine Drainage. Die Drainage wurde postoperativ gezogen, sobald das Sekret serös war und die Fördermenge weniger als $100 \mathrm{ml}$ pro $24 \mathrm{~h}$ betrug. Primärer Endpunkt war das Auftreten einer Anastomoseninsuffizienz, eines Abszesses im Becken oder einer Peritonitis.

M. Schrempf $\cdot$ M. Anthuber

Klinik für Allgemein-, Viszeral- Transplantationschirurgie, Klinikum Augsburg, Augsburg, Deutschland

\title{
Zum Nutzen von Drainagen nach tiefer Rektumanastomose
}

Ergebnisse. Insgesamt 494 Patienten wurden randomisiert, nach Ausschluss von 25 Patienten wurden 236 Patienten in Gruppe A (Drainage) und $233 \mathrm{~Pa}$ tienten in Gruppe B (keine Drainage) ausgewertet. Die Höhe der Anastomose betrug 3,5 $\pm 1,9 \mathrm{~cm}$ ab ano. Die Häufigkeit des primären Endpunkts war in beiden Gruppen gleich (16,1\% mit Drainage vs. $18,0 \%$ ohne Drainage; $p=0,58)$. Die Gruppe mit Drainage unterschied sich nicht von der Gruppe ohne Drainage hinsichtlich der postoperativen Morbidität $(16,1 \%$ vs. $18,0 \%$; $p=0,83)$, der Rate an Revisionseingriffen (16,6\% vs. $21,0 \%)$, der Krankenhausverweildauer (12,2 Tage vs. 12,2 Tage; $p=0,99)$ und der Rate an Stomarückverlagerungen nach 6 Monaten $(80,1 \%$ vs. $77,3 \% ; p=$ $0,53)$.

Diskussion und Fazit. In dieser Arbeit wurde kein Vorteil einer Drainageneinlage nach tiefer anteriorer Rektumresektion nachgewiesen. Gestützt wird dieses Ergebnis durch eine Metaanalyse von 3 randomisierten Studien [2]. Demgegenüber steht das Ergebnis einer zweiten Metaanalyse, die unter Einbeziehung retrospektiver Daten eine geringere Rate an Insuffizienzen und Reinterventionen bei der Verwendung von Drainagen nachweisen konnte [3]. Auch in der größten retrospektiven Arbeit von Peeters et al. war die Insuffizienzrate nach Drainageneinlage signifikant niedriger [1]. Zu beachten ist, dass Peeters et al. im Gegensatz zur hier vorgestellten Arbeit Drainagen ohne Sog verwendeten. Zusammenfassend scheinen die Daten prospektiver Studien darauf hinzuweisen, dass nach unkomplizierter Rektumresektion auf eine Drainageneinlage verzichtet werden kann. Dies gilt insbesondere dann, wenn ein protektives Stoma angelegt wurde. Im Falle einer intraoperativen Eröffnung des Rektums, bei diffuser Blutungsneigung oder bei technisch schwieriger Anastomosenanlage sollte jedoch nicht auf eine Drainage verzichtet werden.

\section{Korrespondenzadresse}

Prof. Dr. M. Anthuber

Klinik für Allgemein-, Viszeral- Transplantationschirurgie, Klinikum Augsburg Stenglinstr. 2, 86156 Augsburg, Deutschland matthias.anthuber@klinikum-augsburg.de

Interessenkonflikt. M. Schrempf und M. Anthuber geben an, dass kein Interessenkonflikt besteht.

\section{Literatur}

1. Peeters KC, Tollenaar RA, Marijnen CA et al (2005) Risk factors for anastomotic failure after total mesorectal excision of rectal cancer. Br J Surg 92:211-216

2. Rolph R, Duffy JM, Alagaratnam S et al (2004) Intra-abdominal drains for the prophylaxis of anastomotic leak in elective colorectal surgery. Cochrane Database Syst Rev. doi:10.1002/ 14651858.cd002100.pub2

3. Rondelli F, Bugiantella W, Vedovati MC et al (2014) To drain or not to drain extraperitoneal colorectal anastomosis? A systematic review and metaanalysis. Colorectal Dis 16:035-42 